\title{
Erratum
}

\section{Relativistic Corrections in Quasi-Free Electro-Disintegration of the Deuteron}

\section{G. Beck and H. Arenhövel}

Institut für Kernphysik, Johannes-Gutenberg-Universität, J.-J.-Becher-Weg 45, D-55099 Mainz, Federal Republic of Germany

[Few-Body Systems 13, 165 (1992)]

In Appendix C, the third line of Eq. (C.9) should read:

$$
\left.+q^{2} \Pi_{j, \lambda} S_{0}^{s m_{s} m_{s}^{\prime}}+2 \lambda k q^{2} \sum_{j^{\prime} \neq j} s_{j^{\prime}} S_{j, j^{\prime}}^{s m_{s} m_{s}^{\prime}}\right]\left\langle\vec{k}_{j} \mid l m_{l}\right\rangle,
$$

where

$$
\begin{aligned}
S_{j, j^{\prime}}^{s m_{s} m_{s}^{\prime}}= & (-)^{s-m_{s}} \sum_{s^{\prime \prime} m_{s}^{\prime \prime}}\left(m_{s}^{\prime \prime}-m_{s}^{\prime}\right)\left(\begin{array}{ccc}
s & 1 & s^{\prime \prime} \\
-m_{s} & m_{s}-m_{s}^{\prime \prime} & m_{s}^{\prime \prime}
\end{array}\right) \\
& \times d_{\lambda, m_{s}-m_{s}^{\prime \prime}}^{1}\left(\theta_{n p}\right) d_{0, m_{s}^{\prime \prime}-m_{s}^{\prime}}^{1}\left(\theta_{n p}\right) S_{1 j^{\prime}}^{s^{\prime \prime} m_{s}^{\prime \prime} m_{s}^{\prime}}\left\langle s\left\|\sigma_{j}^{[1]}\right\| s^{\prime \prime}\right\rangle .
\end{aligned}
$$

Furthermore, Fig. 11 b should be replaced by the following one:

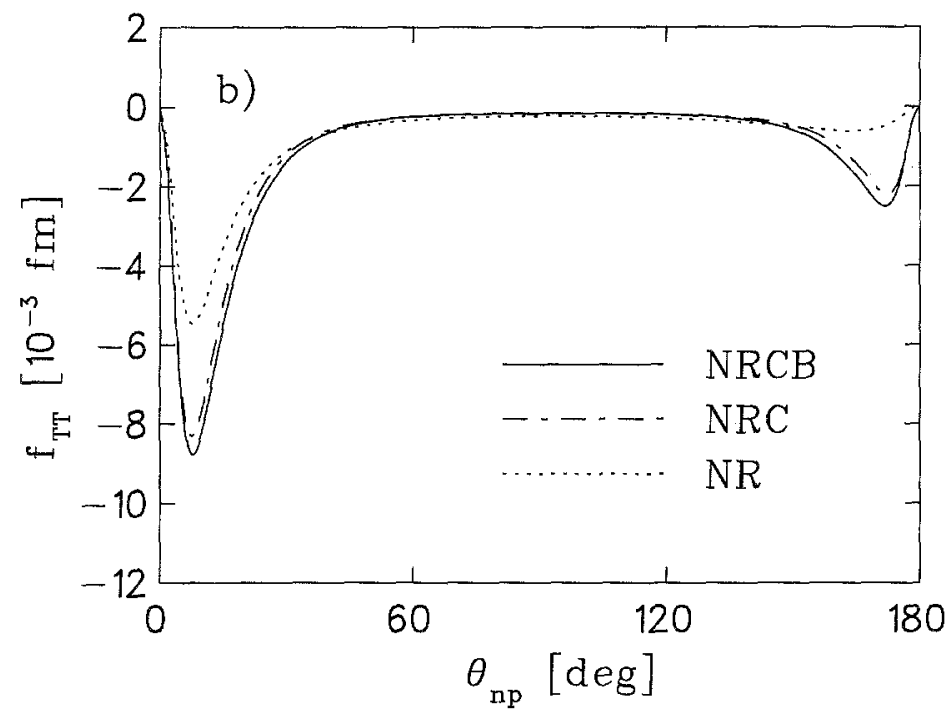

Fig. 11 b

Received July 7, 1993 